\title{
Effects of Essential Oils on Erythrocytes and Hepatocytes from Rats and Dipalmitoyl Phosphatidylcholine-Liposomes
}

\author{
Atsufumi MANABE, Sadao NAKAYAMA and Koji SAKAMOTO \\ Department of Pharmacology. School of Medicine, Showa University. \\ 1-5-8 Hatanodai. Shinagawa-ku, Tokyo 142, Japan
}

Accepted February 2, 1987

\begin{abstract}
The effect of essential oils, eugenol, thymol and menthol, on erythrocytes, hepatocytes, dipalmitoyl phosphatidylcholine (DPPC)-liposomes and surface tension were studied at various concentrations. Maximal inhibition of eugenol, thymol and menthol on the hypotonic hemolysis in rat erythrocytes were observed at a concentration of $2 \mathrm{mM}, 1 \mathrm{mM}$ and $1 \mathrm{mM}$, respectively. Eugenol at $4 \mathrm{mM}$ and thymol at $2 \mathrm{mM}$ caused an acceleration of hypotonic hemolysis. In isolated rat hepatocytes, thymol caused an increase in GOT leakage, but eugenol at $4 \mathrm{mM}$ and menthol at 0.1 and $0.4 \mathrm{mM}$ inhibited the GOT leakage. The leakage of GPT from hepatocytes was inhibited by eugenol at $0.1 \mathrm{mM}$ and 0.4 to $4 \mathrm{mM}$ and menthol at 0.1 to $0.6 \mathrm{mM}$. The inhibition of eugenol and menthol on the LDH leakage in hepatocytes were observed at a concentration of 0.001 to $4 \mathrm{mM}$ and 0.1 . 0.4 and $0.6 \mathrm{mM}$, respectively. Thymol caused no change in GPT and LDH leakage. Eugenol, thymol and menthol indicated a depression of surface tension at a concentration of $0.1 \mathrm{mM}$. The rank by order of surface activity was eugenol >thymol. Eugenol, thymol and menthol depressed the phase-transition temperature of DPPCliposomes. The depression of phase-transition temperature by thymol was greater than that by eugenol and menthol. These results suggest the periapical tissue damage produced by essential oils may be related to membrane lysis and surface activity and that the their tissue penetration may be related to membrane affinity and lipid solubility.
\end{abstract}

Eugenol (2-methoxy-4-allylphenol) is the chemical essence of clove oil and is probably a widely used dental drug in root canal treatment. Clove oil and eugenol have been found to possess the ability as germicide. antiseptic and anodyne (1). Eugenol is a constituent of most root canal sealers and belong more properly under the heading of antiseptic than under germicide. Although eugenol caused an irritation and toxicity on soft tissues $(2,3)$. The irritative effect of eugenol is slightly more potent than that of clove oil (1). Thymol also is a widely used drug in root canal treatment. Thymol has a stronger ablity as germicide and antiseptic than eugenol. It is also more strongly irritating to periapical tissue than eugenol (4). Menthol is the main component of peppermint oil and is prepared in the root canal disinfectants, gargarisma and sedative of dentine (5).

Essential oils has a stronger permeability and irritative ability on the tissue caused by the volatility of these (5). The periapical tissue damage by eugenol and thymol is the inflammation of tissue caused by the irritation, however, the mechanism by which essential oils can produce periapical tissue damage is poorly understood. The present studies were undertaken to clarify the mechanism of tissue damage by essential oils, eugenol, thymol and menthol, using rat erythrocytes, hepatocytes and dipalmitoyl phosphatidylcholine (DPPC)-liposomes.

\section{Materials and Methods}

Drugs: The drugs used in this experiment were eugenol, thymol and L-menthol (Wako 
Pure Chemical Ind., Ltd., Japan). Hepatocytes were stained by using trypan blue (Wako Pure Chemical Ind.. Ltd.. Japan). DipalmitoylL- $\alpha$-phosphatidylcholine (Sigma, U.S.A.) was used for the formation of liposomes.

Hypotonic hemolysis study: Protective and lytic effects of drugs on hypotonic hemolysis were performed by a modified Seeman's method $(6,7)$. Male, 6 week old SpragueDawley rats weighing $160-170 \mathrm{~g}$ were anesthetized with pentobarbital $(40 \mathrm{mg} / \mathrm{kg}$. i.p.), the abdomen was opened, and blood was collected from the inferior vena cava with a heparinized syringe. The blood was centrifuged (255 r.p.m., $15 \mathrm{~min}$ ), and the erythrocyte-rich precipitate was washed three times with isotonic phosphate-buffered saline (PBS: $142 \mathrm{mM} \mathrm{NaCl}$ and $10 \mathrm{mM}$ phosphate, $\mathrm{pH}$ 7.4). The concentration of erythrocytes in PBS was then adjusted to $1 \times 10^{9}$ cells $/ \mathrm{ml}$, using a Thoma's counting chamber, and this erythrocyte suspension was kept at $0^{\circ} \mathrm{C}$ before use. The hypotonic buffer solution which would cause hemolysis of $70 \%$ of the erythrocytes was prepared by adjusting the concentration of sodium chloride in $10 \mathrm{mM}$ phosphate buffer $(\mathrm{pH} 7.4)$ for each erythrocyte suspension. Drugs were first dissolved in dimethylsulfoxide (DMSO) and introduced into the hypotonic buffer solution to produce a concentration of DMSO always less than $1 \%$ volume, and this had been previously found not to affect the degree of hemolysis. Aliquots $(4.3 \mathrm{ml})$ of the hypotonic buffer solution in which various concentrations of each drug were dissolved and aliquots $(0.2 \mathrm{ml})$ of the erythrocyte suspension were separately pre-incubated $\left(37^{\circ} \mathrm{C}, 5 \mathrm{~min}\right)$. They were then mixed in glass test tubes and incubated for $1 \mathrm{hr}$ in a $37^{\circ} \mathrm{C}$ water bath. Following incubation, this suspension was centrifuged (2000 r.p.m., $5 \mathrm{~min})$, and the free hemoglobin in the supernatant was determined at a wavelength of $540 \mathrm{~nm}$ using a Shimadzu spectrophotometer.

Isolation of rat hepatocytes, cytotoxicity and morphological studies: Male. 7 week old Sprague-Dawley rats weighing $220-250 \mathrm{~g}$ were anesthetized with pentobarbital, the abdomen was opened, and the portal vein was cannulated with a 16 guage angioca- theter. Hepatocytes were prepared by a modification of the method of Yasuhara et al. (8). The liver was perfused with $10 \mathrm{mM}$ Hepes buffer ( $\mathrm{pH} \mathrm{7.4)}$ for 10 min. The chest was opened, and the thoracic portion of the inferior vena cava was cannulated via the right atrium. The inferior vena cava was ligated just above the level of the renal veins. The liver was then perfused with Hepes buffer containing $0.05 \%(w / v)$ collagenase and $100 \mathrm{mM} \mathrm{CaCl}_{2}$ at a pressure of 40 $\mathrm{CmH}_{2} \mathrm{O}$ for $45 \mathrm{~min}$. The liver was removed, transferred to a beaker containing Hepes buffer, and split with a blunt spatula. The resulting cell suspension was filtered through two layers of nylon mesh. The pellet was washed twice in Eagle's minimum essential medium (Eagle's MEM: Gibco Oriental Co., Japan) solution and resuspended in this medium to a final concentration of $2 \times 10^{5}$ cells $/ \mathrm{ml}$. When a sample of cells was exposed to $0.25 \%$ trypan blue, 5 to $10 \%$ of them stained, demonstrating 90 to $95 \%$ viability.

Enzyme assay: Drugs were dissolved in DMSO and then diluted in Eagle's MEM. This solution was added to the cell suspensions to attain a final drug concentration of 0.001 to $4 \mathrm{mM}$. Suspensions containing the same final concentration of $1 \%$ DMSO in Eagle's MEM solution were used as controls. The $\mathrm{pH}$ was maintained at 7.4. All procedures were performed using sterile glassware and solutions to reduce microbial contamination. One $\mathrm{ml}$ of drug or control solution was mixed with $1 \mathrm{ml}$ of eagle MEM containing $2 \times 10^{5}$ cells. The suspensions were incubated in culture tubes for $1 \mathrm{hr}$ at $37^{\circ} \mathrm{C}$. The supernatant was assayed for transaminase (GOT. GPT) and lactate dehydrogenase ( $\mathrm{LDH}$ ). Transaminase and $\mathrm{LDH}$ were determined by the colorimetric method using commercial kits (Transaminase-C Test, LDH-C II Test; Wako Pure Chemical Ind., Ltd., Japan) (9. 10).

Morphological observation: Suspensions containing drug or DMSO with hepatocytes were exposed to $0.25 \%$ trypan blue. The stained hepatocytes were transferred to a Thoma's counting chamber. Morphological change and viability of hepatocytes were examined under light microscopy.

Surface tension study: Each drug was 
dissolved in DMSO and then added to 100 $\mathrm{mM}$ phosphate buffer solution to produce concentrations of 0.001 to $10 \mathrm{mM}$. This buffer solution was introduced into the bath of an Acoma Wilhelmy surface balance. Surface tension was measured by the platinum hanging plate method (11) and recorded with a Riken Denki F-3EP X-Y recorder. The final concentration of DMSO was always less than $1 \%$ volume, which was found to have a negligible effect on the surface tension (6).

Phase-transition temperature study: DPPC was dispersed in $10 \mathrm{mM}$ phosphate buffer $(\mathrm{pH}$ 7.4) at a concentration of $1 \mathrm{mM}$ using a Bronson Sonifer All Destruction 200 at a temperature above the phasetransition of DPPC, for $1 \mathrm{hr}$ (6). Essential oils were then dissolved in the DPPC dispersion at 0.001 to $1 \mathrm{mM}$. Aliquots $(3 \mathrm{ml})$ of this solution were introduced into a 10 $\mathrm{mm}$ light-path fluorometry cuvette, which was then placed in the thermostatted doublebeam cuvette compartment of a Hitachi fluorophotometer.

The temperature inside the cuvette was measured to the accuracy of $0.01^{\circ} \mathrm{C}$ with a Takara thermister probe inserted into the cuvette and recorded with a Hitachi $057 X-Y$ recorder. The temperature was increased at a rate of $0.5^{\circ} \mathrm{C} / \mathrm{min}$ by circulating water from a Shimadzu water thermobath. Turbidity was measured by 90 degree light scattering at a wavelength of $440 \mathrm{~nm}$ and recorded by a strip chart recorder. The midpoint between the beginning of the decrease in the scattered light and the point where the scattering reached its plateau was taken as the phasetransition temperature for each concentration of essential oils.

Statistical analysis: The data of hypotonic hemolysis, enzyme leakage in hepatocytes and phase-transition temperature represents the mean \pm S.D. of 3 to 4 experiments. The data of surface tension represents the mean of 2 experiments. Statistical significances were determined using Student's t-test: A $P$ value of $<0.01$ was considered statistically significant.

\section{Results}

Hypotonic hemolysis study: Effects of essential oils on the hypotonic hemolysis are

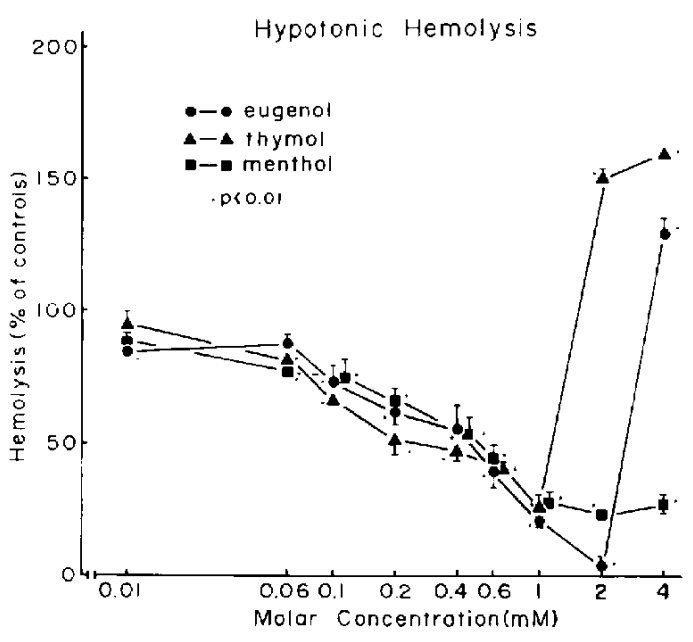

Fig. 1. Effects of essential oils on $70 \%$ hypotonic hemolysis at $\mathrm{pH}$ 7.4. Relative degree of hemolysis is expressed as a percentage of the control value. Each value represents the mean $\pm S . D$. of 4 experiments. *Significantly different from the control $(P<0.01)$.

shown in Fig. 1. The relative degree of hemolysis was expressed as a percentage of the control value of $70 \%$ (the hypotonic solution without a drug would produce $70 \%$ hemolysis).

Eugenol and thymol showed biphasic effects on hypotonic hemolysis. At lower concentrations $(0.1$ to $2 \mathrm{mM})$, eugenol protected erythrocytes against hypotonic hemolysis; and at higher concentration (4 $\mathrm{mM})$, it exerted a lytic effect on the erythrocytes. At 0.06 to $1 \mathrm{mM}$. thymol a!so protected erythrocytes; and at 2 to $4 \mathrm{mM}$, it exerted a lytic effect on the erythrocytes. The concentration which exerted the maximum protection for erythrocytes against $70 \%$ hypotonic hemolysis were about $2 \mathrm{mM}$ and $1 \mathrm{mM}$ for eugenol and thymol, respectively. On the other hand, menthol showed only a protective effect on the erythrocytes at concentrations from 0.06 to $4 \mathrm{mM}$.

Cytotoxicity study: In the hepatocytes, thymol caused an increase in GOT leakage at 0.2 to $4 \mathrm{mM}$, but eugenol at $4 \mathrm{mM}$ and menthol at 0.1 and $0.4 \mathrm{mM}$ inhibited the GOT leakage (Fig. 2). Eugenol (0.1 and 0.4 to $4 \mathrm{mM})$ and menthol $(0.1$ to $0.6 \mathrm{mM})$ caused an inhibition of GPT leakage in the hepatocytes. The leakage of $\mathrm{LDH}$ from hepatocytes was inhibited by eugenol at 

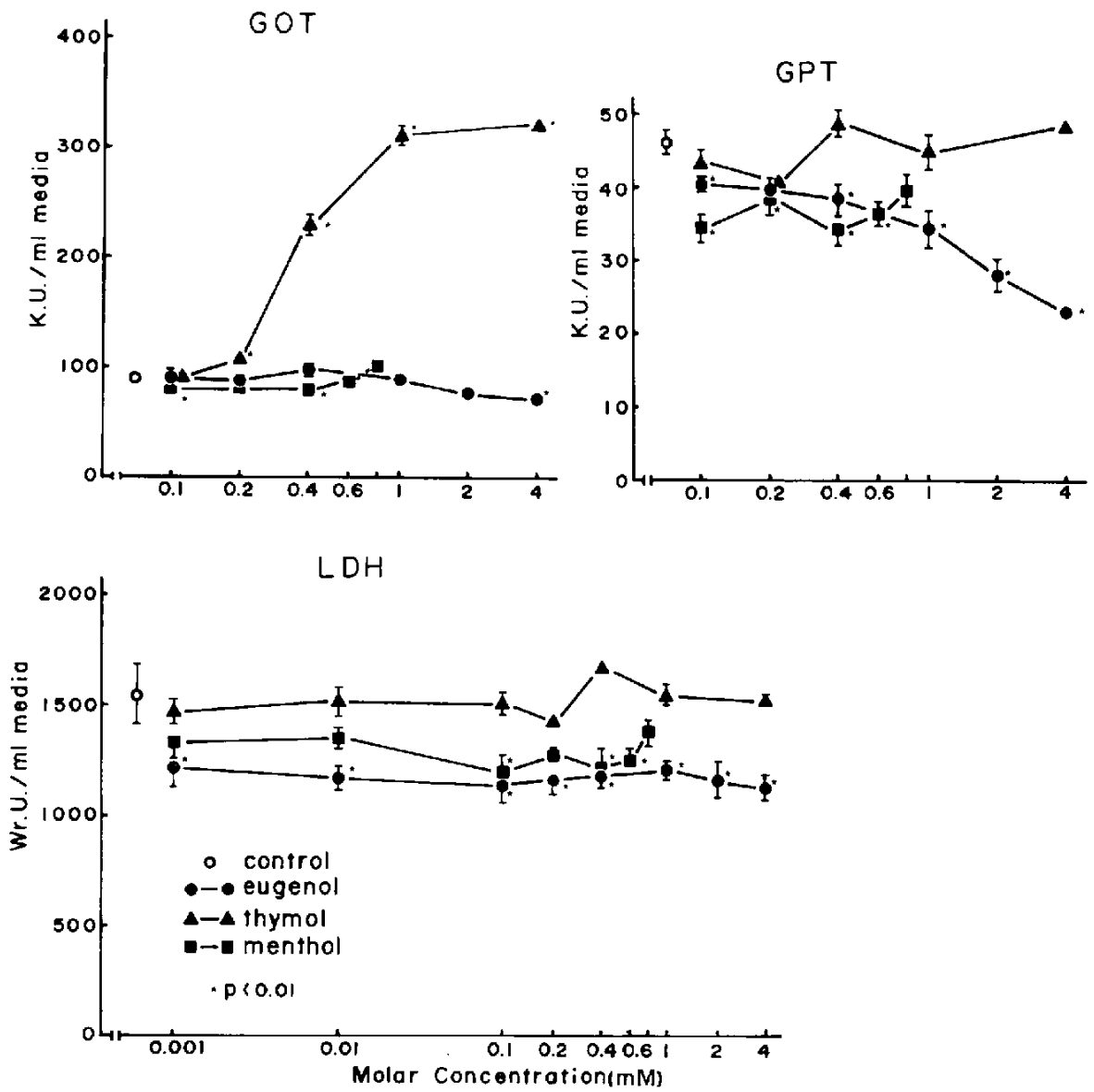

Fig. 2. Effects of essential oils on isolated rat hepatocytes as determined by leakage of GOT, GPT and $\mathrm{LDH}$ into the media. The cells were incubated for $1 \mathrm{hr}$ at $37^{\circ} \mathrm{C}$. Each value represents the mean \pm S.D. of 4 experiments. O: control value (GOT: $92.8 \mathrm{~K} . \mathrm{U} . / \mathrm{ml}$, GPT: $45.9 \mathrm{K.U} . / \mathrm{ml}, \mathrm{LDH}: 1554 \mathrm{Wr} . \mathrm{U} . / \mathrm{ml}$ - ${ }^{*} \mathrm{Sig}$ nificantly different from the control $(P<0.01)$.

0.001 to $4 \mathrm{mM}$ and menthol at $0.1,0.4$ and $0.6 \mathrm{mM}$. Thymol caused no change in GPT and LDH leakage (Fig. 2).

Morphological study: The nucleus of normal hepatocytes was not stained with trypan blue, and the viability of hepatocytes was not lowered by the $1 \mathrm{hr}$ incubation (Fig. $3 a$ ). In the slightly sonicated hepatocytes the cytoplasma was stained with trypan blue and nuclei were lysed. The slight sonication caused a complete loss of hepatocyte viability (Fig. 3b). Eugenol slightly decreased hepatocyte viability. The nucleus of a few hepatocytes were stained with trypan blue, and this observation differed from the sonicated hepatocytes (Fig. 3c). Mor- phological changes in the menthol-added hepatocytes were similar to the changes in eugenol-added ones (Fig. not shown). In the thymol-added hepatocytes, the nuclei were stained with trypan blue, and the viability was markedly lowered (Fig. 3d).

Surface tension study: Effects of essential oils on the surface tension are shown in Fig. 4. The surface tension of phosphate buffer solution without drug was $72 \mathrm{dyne} / \mathrm{cm}$.

Eugenol and thymol decreased the surface tension of the phosphate buffer. At the concentrations of $4 \mathrm{mM}$ for eugenol and $1 \mathrm{mM}$ for thymol, the surface tensions were 38 and 53 dyne $/ \mathrm{cm}$, respectively. Menthol decreased surface tensions of the phosphate 


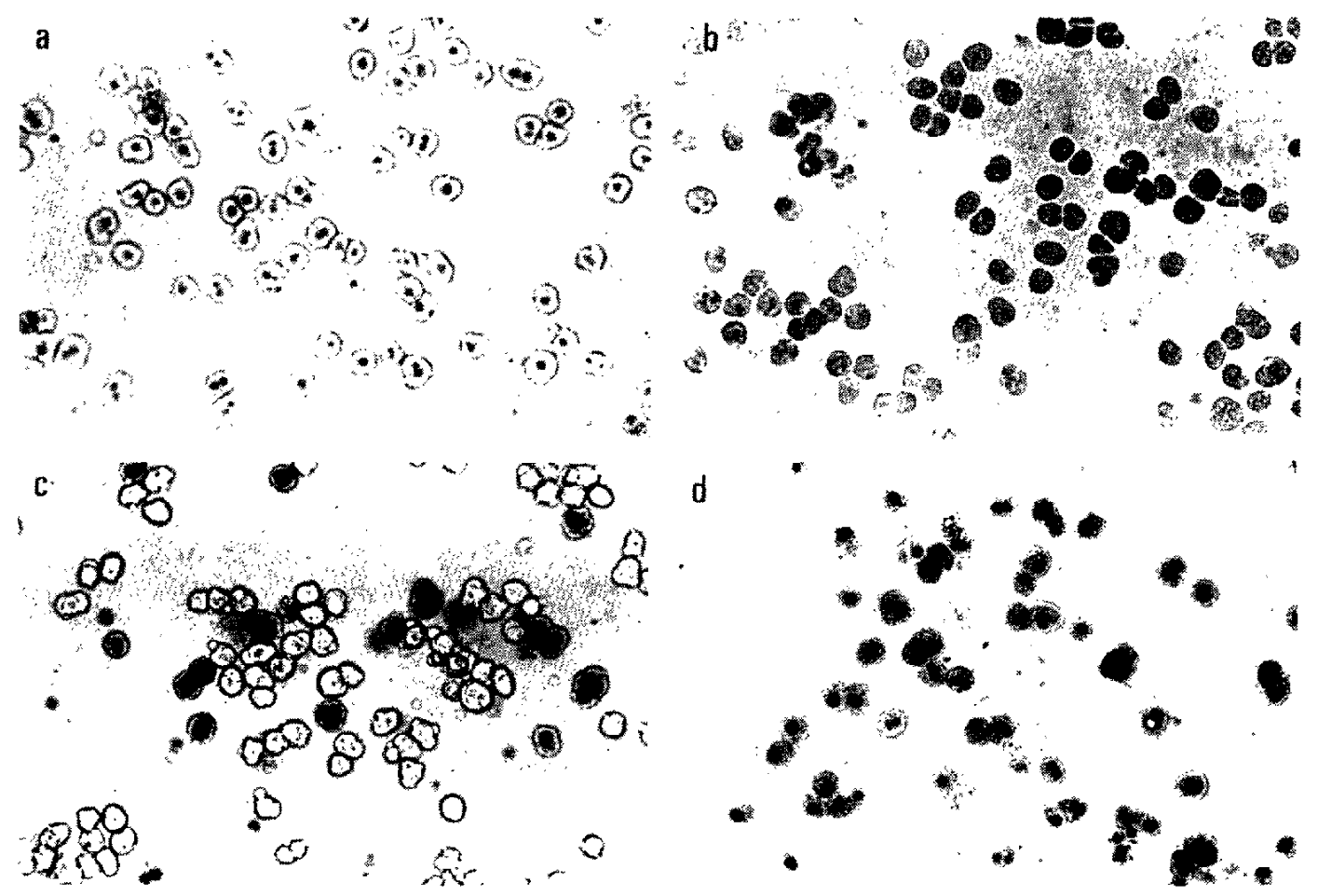

Fig. 3. Effects of essential oils on morphological changes of isolated rat hepatocytes. The cells were incubated with drugs for $1 \mathrm{hr}$ at $37^{\circ} \mathrm{C}$, and cell viability was demonstrated by the staining with trypan blue. a; control, b; slight sonicate, c: $1 \mathrm{mM}$ eugenol, $\mathrm{d}: 1 \mathrm{mM}$ thymol.

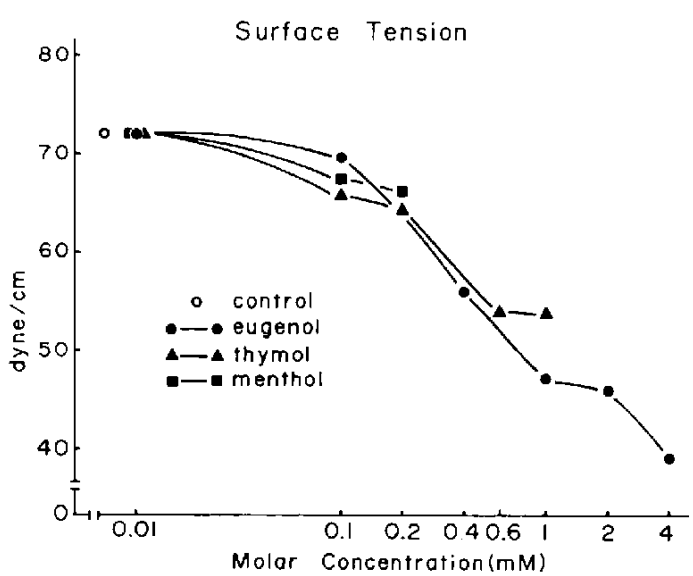

Fig. 4. Effects of essential oils on surface tension at $\mathrm{pH}$ 7.4. Surface tension was measured by the platinum hanging plate method (11) using an Acoma Withelmy surface balance. Each value represents the mean of 2 experiments. 0 : control value (72 dyne/ $\mathrm{cm}$.

buffer from 0.1 to $0.2 \mathrm{mM}$. Because of the low solubility of menthol in the phosphate buffer, we could study the effect of menthol at concentrations above $0.4 \mathrm{mM}$.

Phase-transition temperature study: As shown in Table 1, the phase-transition temperature of DPPC-liposomes without drug was 41.7 to $41.8^{\circ} \mathrm{C}$. Eugenol, thymol and menthol depressed the phase-transition temperature of DPPC-liposomes. Thymol had the greatest membrane fluidity effect since it depressed the phase-transition temperature by $33.3^{\circ} \mathrm{C}$ at $1 \mathrm{mM}$.

\section{Discussion}

The order of effects of germicide and antiseptic using the phenol coefficient was thymol > eugenol $>$ menthol (12). The irritating effect of eugenol and thymol in the periapical tissue was less than that of phenol (13). while the cytotoxic effect of eugenol on the Hela cells was stronger than that of phenol (14). It was also reported that the irritation potential of essential oils on the rabbit skin by intradermal injection was in the following order: eugenol thymol= 
Table 1. Effects of essential oils on phase-transition temperature of dipaimitoyl-L- $\alpha$-phosphatidylcholine-liposomes

\begin{tabular}{cccc}
\hline $\begin{array}{c}\text { Concentration } \\
(\mathrm{mM})\end{array}$ & \multicolumn{3}{c}{ Phase-transition temperature $\left({ }^{\circ} \mathrm{C}\right)$} \\
\hline 0 & Eugenol & Thymol & Menthol \\
0.1 & $41.7 \pm 0.3$ & $41.8 \pm 0.3$ & $41.8 \pm 0.3$ \\
0.2 & $40.6 \pm 0.5$ & $39.9 \pm 1.2$ & $41.0 \pm 0.7$ \\
0.5 & $40.4 \pm 0.1^{*}$ & $38.3 \pm 0.6^{*}$ & $40.5 \pm 0.7$ \\
1.0 & $39.2 \pm 0.1^{*}$ & $36.2 \pm 0.5^{*}$ & $39.4 \pm 0.6^{*}$ \\
\hline
\end{tabular}

Each value represents the mean \pm S.D. of 3 experiments. "Significantly different from the control $(P<0.01)$.

menthol (15).

Both of the effects, protective and lytic, of thymol and eugenol against hypotonic hemolysis of rat erythrocytes indicate that these essential oils are useful drugs for endodontic treatments. Especially, the Iytic effects of thymol and eugenol on the erythrocyte membranes may be related to the irritating activity in the periapical tissue. Menthol showed only a stabilizing effect on the erythrocyte membranes at concentration of 0.06 to $4 \mathrm{mM}$. The potency of membrane stabilizing effects of essential oils on erythrocytes occurred in the following order: eugenol $>$ menthol $=$ thymol.

Thymol caused a marked GOT leakage from isolated rat hepatocytes. In contrast. eugenol and menthol caused only an inhibition of enzyme leakage. The necrosis of hepatocytes after exposure to thymol was confirmed by trypan blue staining as a conventional indicator of cell viability. The concentration of thymol that caused necrosis of hepatocytes was the same as that which caused enzyme leakage. The inhibition of GOT, GPT and LDH leakage by eugenol and menthol may indicate the membrane stabilizing effect of these drugs on the hepatocytes, and the increase of GOT leakage by thymol may suggest membrane lysis. Thymol had a greater ability to cause membrane lysis in erythrocytes and hepatocytes than eugenol. The result seems to be in good agreement with the previous finding that the toxicity of thymol on the cell viability in the guinea pig neutrophils is more potent than that of eugenol. The potency of the membrane Iysis ability of eugenol and thymol on erythrocytes and hepatocytes is very similar to the report of Suzuki et al. (16).

The enzyme release from hepatocytes is regulated by membrane permeability, levels and localization in hepatocytes and molecular weight (17). It was reported that GPT and $\mathrm{LDH}$ in isolated hepatocytes were easily released than GOT (18). Their degrees of release from hepatocytes are not correlated with their molecular weight (GOT: 95000 , GPT: 110000, LDH: 140000). The levels and localization of GOT in hepatocytes are much the same as those of GPT. In the present study, thymol caused an increase of GOT leakage but not GPT and $\mathrm{LDH}$, and it may be correlated with the difference of their enzyme release from the control hepatocytes.

The model membranes of DPPC-liposomes are in a crystalline phase below and liquid crystalline phase above the phase-transition temperature. Thymol depressed the phasetransition temperature more than eugenol and menthol. Depression of the phasetransition temperature of DPPC-liposomes by essential oils indicates an increase in the fluidity of phospholipid bilayer membranes (6. 19). The concentrations of essential oils causing depression of phase-transition temperature were consistent with the concentration caus ng membrane stabilizing effects on hypotonic hemolysis in erythrocytes. The potency of the surface tension reducing effect of the essential oils was in the following order: eugenol >thymol. A reduction of surface tension by eugenol and thymol indicates their affinity for the membrane. The biphasic effects, protective and Iytic, of eugeno! and thymol on the hypotonic hemolysis of erythrocytes may be related to the membrane affinity of these drugs. 
Therefore, the membrane stabilizing action occurs with a moderate uptake of drugs into the erythrocytes, and the membrane lytic action is caused by the expansion of erythrocytes by the excessive uptake of drugs.

A positive correlation between membrane stabilizing effects in erythrocytes, depression of phase-transition temperature in DPPCliposomes and reduction of surface tension has been observed in many studies $(8,20$. 21). We obtained a positive correlation between these results by the essential oils. Furthermore, we obtained a positive correlation between membrane lysis, depression of phase-transition temperature and reduction of surface tension, which is consistent with the results reported in previous studies $(6,22,23)$. In the present study, eugenol and thymol showed a positive correlation between the hemolysis of erythrocytes in higher concentrations, depression of phase-transition temperature and reduction of surface tension. Especially, the increase in enzyme leakage and in necrosis of hepatocytes by thymol was related to the increase in fluidity of phospholipid bilayer membranes and reduction of surface tension.

The inhibiting effects of essential oils on the enzyme leakage from hepatocytes were dose-independent in comparison with effects of acceleration of enzyme leakage in hepatocytes and hypotonic hemolysis in erythrocytes. These results suggested that isolated rat hepatocytes as a biomembrane model was more suitable for the examination of membrane lysis than for examining membrane stability (8, 20,22).

The results of membrane stability and reduction of phase-transition temperature and surface tension in the in vitro cytotoxicity indicate the stabilizing effects of essential oils on hepatocytes and erythrocytes. The results of membrane lysis indicate that the hepatocytes and erythrocytes were damaged by essential oils.

The requirements of a root canal disinfectant are as follows: 1) an effective germicide and fungicide, 2) nonirritating, 3) stable in solution, 4) capable of penetrating the tissue deeply. Eugenol may be more suitable more than thymol as a canal dis infectant.
These results can be explained in terms of the potency in stability, lysis and affinity to membranes. These results also suggested that the mechanism of periapical tissue damage by eugenol and thymol might be related to their abilities to cause membrane lysis in higher concentrations, and that the tissue penetration of these drugs may be related at least in part to their membrane affinity and lipid solubility.

\section{References}

1 Grossman, L.1.: Disinfection of the root canal. In Endodontic Practice 10th., Edited by Grossman. L.I., p. 252-253, Lea and Febiger. Philadelphia (1981)

2 Kozam, G. and Mantell, G.M.: The effect of eugenol on oral mucous membrane. J. Dent. Res. 57, 954-957 (1978)

3 Webb, J.G., Jr. and Bussell, N.E.: A comparison of the inflammatory response produced by commercial eugenol and purified eugenol. J. Dent. Res. 60, 1724-1728 (1981)

4 Asai, Y., Morimoto, M., Yamagishi, A., Nakamura, Y., Maeda, K., Ishikawa, T. and Sekine, N.: Clinical pathological study of thymol on indirect pulp capping effect. J. Tohoku Dental College Soc. 67, 387-401 (1967) (in Japanese)

5 Ogura, H.: Modern Dental Pharmacology, Edited by Ogura. H. and Ogura, Y., p. 198-199. Ishiyaku Shutsupan. Tokyo (1979) (in Japanese)

6 Arakawa, K., Tonooka, M., Goto, H. and Sakamoto, K: Membrane stabilizing action of NCO650 and its congeners. Japan. J. Pharmacol. 36, 311-318 (1984)

7 Seeman, P.: A method for distinguishing specific from nonspecific himolysis. Biochem. Pharmacol. 15, 1767-1774 (1966)

8 Yasuhara, H., Dojovne, C.A., Ueda, I. and Arakawa, K.: Hepatotoxicity and surface activity of tricyclic antidepressants in vitro. Toxicol. Appl. Pharmacol. 47, 47-54 (1979)

9 Ohkawa, J., Miyoshi, Y., Matsuura, S. and Misaki, H.: A new colorimetric method for the determination of transaminase activity using pyruvate oxidase. Japan. J. Clin. Pathol, 26, Supp. 70 (1978) (in Japanese)

10 Babson, A.L. and Phillips, G.E.: A rapid colorimetric assay for serum lactic dehydrogenase. Clin. Chim. Acta 12, 210-215(1965)

11 Lin, H., Ueda, I., Lin, S.H., Shieh, D.D., Laymaa $H$. and Etring, $H$. .: Surface activities of tertiary amine local anesthetics at air/water interface in 
the presence and absence of phospholipid monolayers. Blochim. Biophys. Acta 598, 51-65 (1980)

12 Takagi, N.: Dental Pharmacology. Edited by Yamamoto. I., Tsujimoto, A. and Inoki, R., p. 26-74, Ishiyaku Shutsupan, Tokyo (1971) (in Japanese)

13 Hasegawa, M.: Endodontic Practice. p. 197. Ishiyaku Shutsupan. Tokyo (1975) (in Japanese)

14 Engström, B. and Spangberg, L.: Studies on root canal medicaments, 1. Cytotoxic effect of root canal anticeptics. Acta Odontol. Scand. 25, $77-$ 84 (1967)

15 Grossman, L.I, and Lally, E.T.: Assessment of irritation potential of essential olls for root canal cement. J. Endodontics 8, 208-212 (1982)

16 Suzuki, Y., Sugiyama, K. and Furuta, H.: Eugenolmediated superoxide generation and cytotoxicity in guinea pig neutrophils. Japan. J. Pharmacol. 39, 381-386 (1985)

17 Suzuki, H:: Enzymatic diagnosis-GOT, GPT. Clinic All Round 26, 1272-1275 (1977) (in Japanese)
18 Ichihara, A. and Shirai, A.: The release mechanism of serum enzymes. Metabolism and Disease 5 , 94-99 (1968) (in Japanese)

19 Ueda, I., Tashiro, C. and Arakawa, K.: Depression of phase-transition temperature in mode! cell membrane by local anesthetics. Anesthesiology 46, 327-332 (1977)

20 Matsuo, H.: Effects of tricyclic drugs on erythrocytes and isolated hepatocytes. J. Showa Med. Assoc. 41, 137-145 (1981) (Abs. in English)

21 Salhab, A.S., Yasuhara, H. and Dojovne, C.A.: Surface activity, cellular uptake and cytotoxicity of tricyclic psychoactive drugs in vitro. Biochem. Pharmacol. 28, 1713-1718 (1979)

22 Dojovne, C.A.: Liver cell culture toxicity and surfactant potency of erythromycin derivatives. Toxicol. Appl. Pharmacol. 32, 11-20 (1975)

23 Dojovne, C.A., Yasuhara, $H$. and Ueda, I.: Relationship among hepatotoxicity, surface pressure and cellular uptake of bile acids. Gastroenterology 46, 327-332 (1977) 